\title{
Using Deep Learning to Deconvolute Complex Spectra for Hyperspectral Imaging Applications
}

Samantha Rudinsky ${ }^{1}$, Yu Yuan ${ }^{1}$, Francis B. Lavoie², Raynald Gauvin'1, Ryan Gosselin², Nadi Braidy², Nicolas Piché ${ }^{3}$, Mike Marsh ${ }^{4}$

${ }^{1}$. Department of Materials Engineering, McGill University. Montreal, Canada.

2. Department of Chemical Engineering and Biotechnological Engineering, Université de Sherbrooke. Sherbrooke, Canada.

3. Object Research Systems. Montreal, Canada.

4. Object Research Systems. Denver, USA.

X-ray energy dispersive spectroscopy (EDS) for materials characterization is one of most widespread analytical methods. When EDS is applied in rastering spatial patterns, the aggregate spectra yield hyperspectral images (HSI) that provide a quick and precise interpretation of the elemental spatial distribution of nanomaterials. However the long dwell time required for acquisition of each spectrum makes the experiments highly resource-intensive. We discuss here a Deep Learning approach that uses neural networks to deconvolute overlapping spectra having a weak signal-to-noise ratio, thereby making more precise and definite interpretation possible with shorter imaging times and electron doses.

When the sample probe acquires a single spot, the emitted x-ray photons are observed and binned in an energy spectrum. Depending on spot size and beam energy, the detected x-rays may come from electron-sample interaction of multiple elements on the surface and at depth. In such a case, peak overlap can occur and limit our ability to separate and quantify the individual elemental abundance. Straightforward spectral interpretation such as measuring peak-heights, integrating under peaks, and even computing ratios of observed peak integrals are inadequate for resolution of complicated materials. More sophisticated multivariate statistical solutions such as principal components analysis (PCA) [1] take advantage of x-ray counts in all recorded bins in order to find a linear combination of bins that produce the best discrimination between competing materials. Such approaches improve chemical resolution but are still insufficient for many complicated materials: they may require reference spectra (linear least-square fitting), show unreal spectral response (PCA) or fail with low signal-to-noise ratio (non-negative matrix factorization).

Deep Learning, the application of deep neural networks, presents advantages over multivariate statistical techniques and other machine learning approaches because their implicit mathematical models permit extensive nonlinearity; they exhibit great potential to learn from examples, and there are many successes where they are capable of correctly labeling input signals. Here, we used neural networks to identify the elements abundance in a HSI by training a neural network with Monte-Carlo simulated training set. 
The first stage of a Deep Learning solution is the training of the neural network where many example cases are evaluated and used to update the mathematical model. The second stage is inference whereby the model is presented with an unknown test and asked to make a prediction (in this case, to identify the compounds responsible for the observed spectrum). Once a model is trained over a representative set of known examples, it may be used countless times for the prediction on unknowns.

We implemented our Deep Learning solution as an extension to Dragonfly, a software platform for image analysis that is free for non-commercial use. Dragonfly has previously been shown useful for the inspection and analysis of HSI and other high-dimensional signals [2]. Our extension permits the user to pair HSI with their corresponding chemical maps. These pairings constitute the examples used for training data. Users can prepare these training data experimentally by analyzing samples where the chemical composition is already known with high spatial accuracy. Or they may create training data by simulation of X-ray spectra from first principles, using tools such as MC X-ray [3]

The trained models show high predictive power. Figure 1 shows the simulated chemical map along with the interpreted map as predicted by the trained 1-dimensional convolutional neural network. The model was trained with simulated EDS spectra representing various concentrations of $\mathrm{Al}, \mathrm{Ti}, \mathrm{V}$ and Fe.

Being able to interpret noiser, lower dwell-time EDS experiments is important for high-throughput chemical mapping. Our solution here, offered as a Dragonfly extension, makes it easy for users to train new models, and it is highly portable since users can share their trained models in the Infinite Toolbox online repository.

\section{References:}

[1] P.G. Kotula, et al., Microscopy and Microanalysis 9 (2003), p. 1-17.

[2] N. Piché, et al., Microscopy and Microanalysis 24 (Suppl 1) (2018), p. 560-561.

[3] R. Gauvin, et al., Microscopy and Microanalysis 15 (Suppl 2) (2009), p. 488-489.
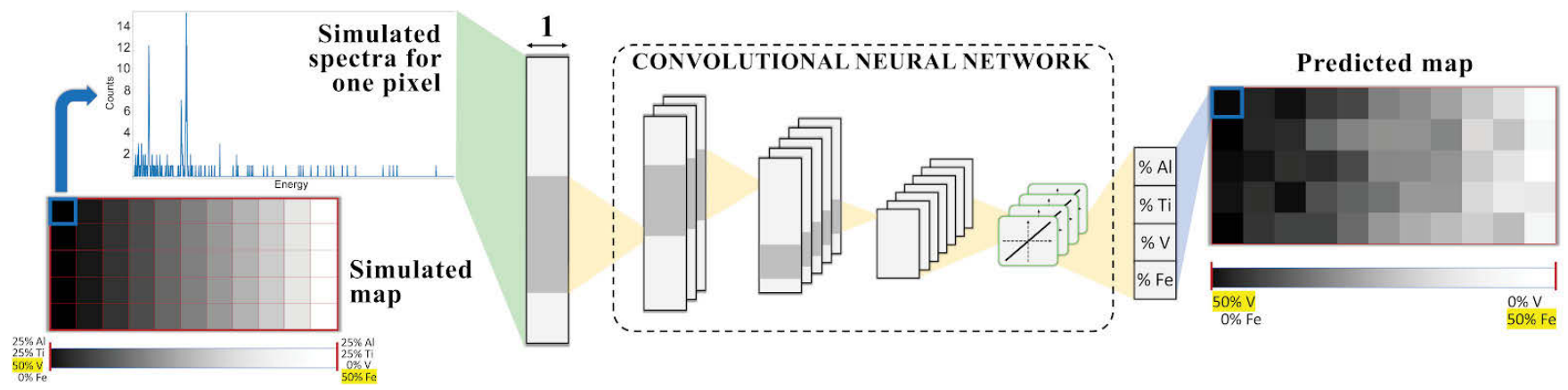

Figure 1. Schematic of mapping of spectrum to 1D convolutional neural network. 